\title{
Nanoplastics decrease the toxicity of a complex PAH mixture but impair mitochondrial energy production in developing zebrafish
}

\author{
Rafael Trevisan ${ }^{1,{ }^{*}, \text { Ciara Voy }}{ }^{1}$, Shuxin Chen ${ }^{2}$, Richard T. Di Giulio ${ }^{1}$ \\ ${ }^{1}$ Nicholas School of the Environment, Duke University, Durham, NC 27708, USA \\ ${ }^{2}$ North Carolina School of Science and Mathematics, Durham, NC 27705, USA
}

\begin{abstract}
Plastics are recognized as a worldwide threat to the environment, possibly affecting human health and wildlife. Small forms of plastics such as micro and nanoplastics can interact with other organic contaminants, potentially acting as chemical carriers and modulating their toxicity. In this study, we investigated the toxicity of polystyrene nanoparticles (Nano-PS) and a real-world environmental PAH mixture (Elizabeth River Sediment Extract - ERSE, comprised of 36 detected PAHs) to zebrafish embryos and larvae. Embryos were exposed to Nano-PS $(0.1-10 \mathrm{ppm})$ or ERSE $(0.1-5 \% \mathrm{v} / \mathrm{v}$, equivalent to $\Sigma \mathrm{PAH} 5.07-25.36 \mathrm{ppb})$, or co-exposed to a combination of both. Larvae exposed to Nano-PS did not exhibit developmental defects, while larvae exposed to ERSE $(2-5 \%)$ showed classic signs of PAH toxicity such as heart malformation and deformities in the jaw, fin, and tail. ERSE (5\%) also impaired vascular development in the brain. When coexposed, Nano-PS decreased the developmental deformities and impaired vascular development caused by ERSE. This was strongly correlated to the lower PAH bioaccumulation detected in the co-exposed animals (whole larvae, as well as the yolk sac, brain, and heart). Our data suggest that PAHs are sorbing to the surface of the Nano-PS, decreasing the concentration, uptake, and toxicity of free PAHs during the exposure. Such sorption of PAHs increases the agglomeration rate of Nano-PS during the exposure time, potentially decreasing the uptake of Nano-PS and associated PAHs. Despite that, similar induction of EROD activity was detected in animals exposed to ERSE in the presence or not of Nano-PS, suggesting that enough PAHs were accumulated in the organisms to induce cellular defense mechanisms. Nano-PS exposure (single or combined with ERSE) decreased the mitochondrial coupling efficiency and increased NADH production, suggesting and impairment on ATP production accompanied by a compensatory mechanism. Our data suggest that nanoplastics can sorb contaminants and potentially decrease their uptake due to particle agglomeration. Nanoplastics also target and disrupt mitochondrial energy production and act as vectors for the mitochondrial uptake of sorbed contaminants during embryonic and larval stages. Such negative effects of nanoplastics on energy metabolism and efficiency could be
\end{abstract}

\footnotetext{
*Corresponding author: rafael.trevisan@ duke.edu.

Declaration of Interests

The authors declare no competing interests.

Supporting Information

Additional information regarding the protocol for measuring oxygen consumption rates, the chemical composition of the real-world environmental mixture of PAHs (ERSE), ATR-FTIR, DLS and zeta potential analysis of the polystyrene nanoparticles (Nano-PS), survival rates to single or combined exposures of Nano-PS and ERSE, mitochondrial bioenergetics data, and mitochondrial mass data (PDF).
} 
detrimental under multiple-stressors exposures and energy-demanding scenarios, what remains to be validated.

\section{Graphical abstract}

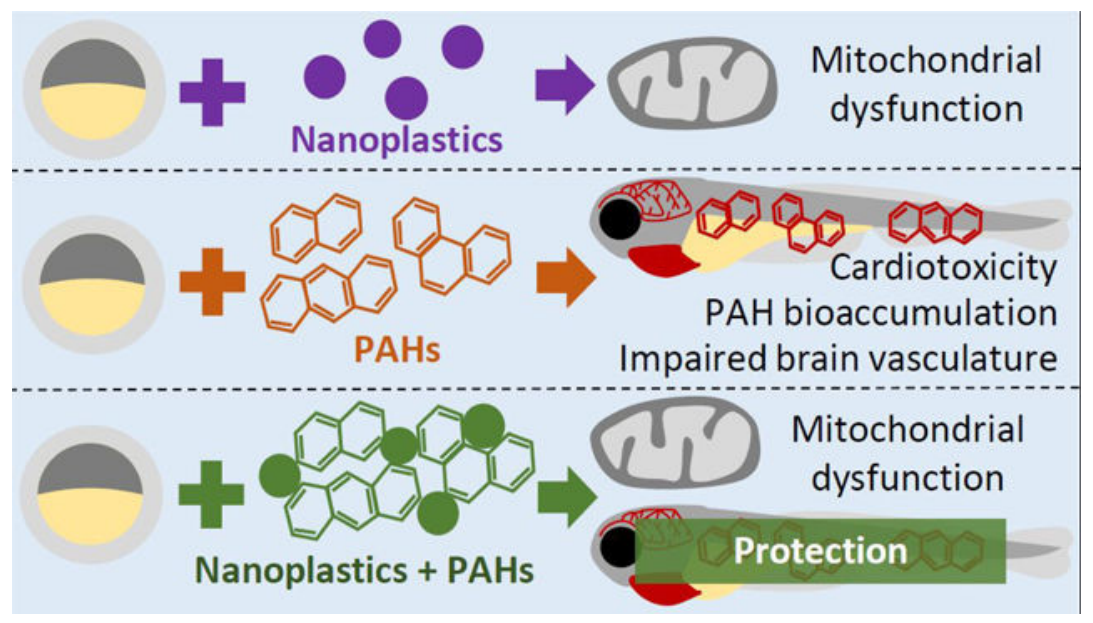

\section{Introduction}

Plastic pollution has recently received worldwide attention as plastics are increasingly recognized as an environmental threat ${ }^{1}$. Inexpensive cost and a broad range of properties are key factors for widespread plastic use and applications, yet the disposal of plastic products - especially single-use plastics - is a major challenge to our modern society. Despite their durability and long lifetime in the environment, weathering and degradation of plastics can cause the release of smaller particles, called microplastics (smaller than $5 \mathrm{~mm}$ ) or nanoplastics (smaller than $1 \mu \mathrm{m})^{2}$. Microplastics have been found worldwide in all aquatic compartments and were found to be ingested by a variety of aquatic organisms, most of the time with adsorbed environmental contaminants ${ }^{1,3}$. However, we still lack information about the fate and levels of nanoplastics in the environment due to the size limit for their recovery in complex environmental samples and detection ${ }^{2,3}$. This is of great concern as the abundance and the uptake of plastic particles by different trophic levels is expected to increase as the particle size decreases, suggesting that nanosized plastic particles might be of even higher concern in the environment ${ }^{3}$.

With the growing body of evidence about the hazards of microplastics, nanoplastics have now appeared as another potentially toxic plastic pollutant. Polystyrene nanoparticles (Nano-PS) have been used as a model type of nanoplastic, and the data obtained so far are of great concern. Nano-PS can have longer gut retention time than polystyrene microparticles ${ }^{4}$, exposure of primary producers such as algae to Nano-PS can lead to the trophic transfer of nanoplastics to crustaceans and fish ${ }^{5}$, and Nano-PS can be passed down to offspring 6,7 Exposure of zebrafish (Danio rerio) embryos to Nano-PS causes the accumulation of nanoplastics mostly in lipid-rich areas such as the yolk sac, with subsequent transport to the digestive system, heart, and brain ${ }^{8}$. The toxicity of Nano-PS is variable but has been 
associated with oxidative stress, immunotoxicity, neurotoxicity, and energy dysfunction in aquatic species $^{7-10}$.

Previous data from our group (Di Giulio laboratory, Duke University) have shown that 34 $40 \mathrm{~nm}$ Nano-PS accumulates in zebrafish embryos through waterborne exposures or maternal transfer, and can affect the antioxidant system, the developing heart, and larval locomotor activity 7,8 . As many nanoplastics are made of hydrophobic polymers and have large surface area to volume ratios, they can act as a significant source for sorption of organic contaminants ${ }^{11,12}$. This is of great concern as they can cause a "Trojan Horse" effect, transferring other environmental contaminants not only to directly exposed organisms and environments but also indirectly through food webs and long-range transport of particles ${ }^{1}$. This carrier effect also points to the likely interaction of nanoplastics with cocontaminants such as polycyclic aromatic hydrocarbons (PAHs), pesticides, polychlorinated biphenyls (PCBs), and many 'priority pollutants', increasing their concentrations to levels 100 times higher than those in sediments and 1 million times higher than those in seawater 13. This effect has the potential to increase the chemical uptake and bioaccumulation rates of such co-contaminants ${ }^{13}$, and possibly modulate molecular targets and modes of action of plastic particles and associated compounds.

This study was designed to analyze if the ability of organic contaminants to adsorb to nanoplastics could interfere with the uptake and toxicity of a real-world complex PAH mixture, focusing on developmental toxicity and mitochondrial bioenergetics. For this aim, we used $44 \mathrm{~nm}$ non-functionalized Nano-PS as a model nanoplastic, for which we have previously described the uptake, bioaccumulation, and toxicity in zebrafish embryos, larvae, and adults 7,8 .

\section{Material and Methods.}

\subsection{Plastic nanoparticles and real-world environmental PAH mixture.}

Nano-PS was purchased from Bangs Laboratories, Inc. (Fishers, IN, USA) as a 10\% stock solution (w:v) with a nominal diameter of $44 \mathrm{~nm}$. Additionally, the stock solution contained $0.1 \%$ sodium dodecyl sulfate (SDS) and $0.05 \%$ sodium azide. Prior to each experiments, an aliquot of stock solution was prepared as $0.1 \%$ Nano-PS in 30\% Danieau's medium (17.4 $\mathrm{mM} \mathrm{NaCl}, 0.21 \mathrm{mM} \mathrm{KCl}, 0.12 \mathrm{mM} \mathrm{MgSO}_{4}, 0.18 \mathrm{mM} \mathrm{Ca}\left(\mathrm{NO}_{3}\right)_{2}$, and $1.5 \mathrm{mM}$ HEPES at a pH 7.6) ${ }^{14}$ The aliquot was sonicated for $60 \mathrm{~s}$ at $50 / 60 \mathrm{~Hz}$ using NEY Ultrasonic Cleaner ULTRASONIK 2QT/H (Barkmeyer Division Yucaipa, Ca, USA) and used for further experiments to achieve the final concentration of $1 \mathrm{ppm}$ (physicochemical characterization) or $10 \mathrm{ppm}$ (toxicity assays).

The polystyrene composition of Nano-PS was characterized by ATR-FTIR (Nicolet 8700, Thermo Fisher Scientific, Waltham, MA, USA) in samples previously filtered to remove sodium azide and sodium dodecyl sulfate (300,000 MWCO), and air-dried (Fig. S2). NanoPS were characterized using dynamic light scattering (DLS) (Zetasizer Nano, Malvern Instruments Ltd., Malvern, UK). The hydrodynamic diameter and zeta potential of Nano-PS were assessed in 30\% Danieau, in a total of 3 samples. The calculated values for freshly prepared Nano-PS were $44.73 \mathrm{~nm}$ (hydrodynamic diameter) and $-38.0 \mathrm{mV}$ (zeta potential) 
(Fig. S3). Nano-PS were also analyzed after $24,48,72$, or 96 hours of incubation in $30 \%$ Danieau at $28{ }^{\circ} \mathrm{C}, 60 \mathrm{rpm}$, and 14:10 hours light/dark cycle, in the presence or not of a realworld environmental mixture of PAHs (described below), and the results are shown in Fig. S3. All samples were diluted 10 times prior to DLS and zeta potential analysis.

A sediment extract from the Atlantic Wood Industries Superfund site, located in the Elizabeth River (VA, USA) was previously collected, processed and characterized by our laboratory ${ }^{15}$. This sediment extract, hereafter known as Elizabeth River Sediment Extract (ERSE), is a real-world environmental mixture of water and suspended solids, with a total PAH content of 5,073 ng/mL PAHs as result of 36 different analyzed PAHs ${ }^{15}$. These PAHs originated from creosote used for wood treatment during the $20^{\text {th }}$ century in that area. The ERSE was further diluted in 30\% Danieau to achieve concentrations ranging from $0.1 \%$ to $5 \%$ ERSE (equivalents of $5.07 \mathrm{ng} / \mathrm{ml}$ to $253.65 \mathrm{ng} / \mathrm{mL} \Sigma$ PAHs). The chemical composition of the ERSE solution is described in the Supplementary Material (Table S1).

\subsection{Embryonic exposures.}

Experiments utilized laboratory-reared wild-type zebrafish (Ekkwill Waterlife Resources; Ruskin, FL), or the transgenic lines Tg(Flk1:EGFP) (a generous gift from Dr. David Tobin, Duke University) and Tg(MLS-EGFP) (a generous gift from Dr. Seok-Yong Choi, Chonnam National University, and Dr. Weibin Zhou, Duke University). Animals were maintained in a recirculating AHAB system (Aquatic Habitats, Inc., Apopka, FL, USA) on a 14:10 h light/ dark cycle. Water quality was maintained at $27-29^{\circ} \mathrm{C}, \mathrm{pH} 7.0-8.0$, using carbon-filtered water supplemented with commercial sea salts (60 mg per liter; Instant Ocean, Foster \& Smith, Rhinelander, WI, USA). The fish were fed twice daily with brine shrimp (INVE Aquaculture, Inc., Salt Lake City, UT, USA) in the morning and Zeigler's Adult Zebrafish Complete Diet (Aquatic Habitats, Inc.) in the afternoon. Breeding crosses (multiple tanks with 3 females: 2 males) were set at 5 PM, and eggs were collected the following morning within $1 \mathrm{~h}$ of spawning between 9 and $10 \mathrm{AM}$ and kept in $30 \%$ Danieau's medium at $28^{\circ} \mathrm{C}$. All zebrafish procedures and exposures were approved by the Duke University Institutional Animal Care and Use Committee (A139-16-06).

Zebrafish embryos at 6 hours post fertilization (hpf) (shield stage) were inspected by light microscopy for proper development and transferred to glass petri dish at a final density of 1 embryo/mL in 30\% Danieau. All exposures were carried out with 10 embryos per petri dish, but the number of Petri dishes used and biological replicates analyzed varied for each assay and are detailed in the following subsections.

Treatment solutions included Nano-PS ( $0.1 \mathrm{ppm}, 1 \mathrm{ppm}$ or $10 \mathrm{ppm})$, ERSE $(0.1 \%, 0.5 \%$, $1 \%, 2 \%$ and $5 \%$ ) or a combination of both Nano-PS and ERSE (10 ppm Nano-PS $+1 \%, 2 \%$ or $5 \%$ ERSE). The exposure started by the addition of freshly prepared Nano-PS, an aliquot of the stock (100\%) ERSE solution, or both, to petri dish containing 30\% Danieu and zebrafish embryos. The exposure dishes were immediately transferred to an Innova 4300 incubator orbital shaker (New Brunswick, Edison, NJ, USA) and kept at $28{ }^{\circ} \mathrm{C}$ and $60 \mathrm{rpm}$, on a 14:10 h light/dark cycle until the end of the exposure ( $24 \mathrm{hpf}, 48 \mathrm{hpf}$ or $96 \mathrm{hpf}$, depending on the assay). The use of an orbital shaker aimed to improve the interaction between Nano-PS and ERSE during the exposures, as PAHs are better mixed in the exposure 
medium by the use of a gentle mixing ${ }^{16}$. This mixing speed yield no detectable effects on development. The exposure medium was not changed or refreshed during the whole experiment (static exposure).

For all experiments, the control group contained $0.00001 \%$ SDS and $0.000005 \%$ sodium azide (concentrations equivalent to the $10 \mathrm{ppm}$ Nano-PS exposure group). For single exposure or co-exposures, all groups had the SDS and sodium azide concentrations adjusted to these values.

\subsection{Survival and deformities assessments.}

Each experiment consisted of 2 Petri dishes per group, with a total of 3 independent experiments. Mortality was determined as the lack of heartbeat or blood flow circulation. The survival and deformities rates were calculated for each individual petri dish at $96 \mathrm{hpf}$ by light microscopy $(n=6)$. The deformities assessed included pericardial edema, yolk sac edema, curved tail, jaw deformities, fin deformities, and string heart.

\subsection{Heartbeat rate and ethoxyresorufin-O-deethylase (EROD) activity.}

For the analysis of heartbeat rate and EROD activity, two Petri dishes per group were used and 5 embryos were randomly chosen per dish per assay.

At $48 \mathrm{hpf}$, embryos were analyzed by light microscopy and the heartbeat was counted over 15 seconds. Data were normalized per minute, and the experiment was repeated 2 times $(n=$ 20).

At $96 \mathrm{hpf}$, larvae were individually transferred to a 384-well solid white microplate, with the addition of $100 \mu \mathrm{L} 30 \%$ Danieau and $10 \mu \mathrm{L}$ of $15 \mu \mathrm{M}$ ethoxyresorufin. The fluorescence was measured in vivo using a FLUOstar Optima (BMG Labtech Inc., Cary, NC, USA) at 530/10 $\mathrm{nm}(\mathrm{ex})$ and $590-10 \mathrm{~nm}(\mathrm{em})$ in cycles of $30 \mathrm{~min}$ over $8 \mathrm{~h}$, at $28^{\circ} \mathrm{C}$. The fluorescence values at $8 \mathrm{~h}$ were subtracted from the blank (30\% Danieau) and normalized to the control group. The assay was adapted from a previously published protocol ${ }^{17}$, and the experiment was repeated 3 times $(n=30)$.

\subsection{PAH bioaccumulation.}

Each experiment consisted of 1 petri dish per group. At 96 hpf, 4 larvae were randomly selected and analyzed by fluorescence microscopy. To prevent the interference of pigmentation on fluorescence imaging, $0.2 \mathrm{mM}$ phenylthiourea (PTU) was added to each petri dish at $24 \mathrm{hpf}$ to block pigment formation. Animals were anesthetized in $200 \mathrm{mg} / \mathrm{ml}$ tricaine (prepared in 30\% Danieau) and transferred to a concave microscope slide with methyl cellulose (3\%). Images were acquired using a Zeiss Axioskop (Carl Zeiss, Oberkochen, Germany) with 480/30 nm (em) and 535/40 nm (ex) filters. Fluorescence was quantified using ImageJ software 17 and expressed as fold change normalized to the control group ${ }^{18}$. The experiments were repeated 3 times $(n=12)$. 


\subsection{Blood vessel formation.}

For this assay, $\operatorname{Tg}(F l k l$ :EGFP) embryos were used for the exposures. This transgenic line expresses enhanced green fluorescence protein (EGFP) in vascular endothelial cells during later stages of the development. The experiments were carried out as described in section 2.5 , except that during image analysis, the threshold function was used to detect the fluorescence related only to the blood vessels, and the associated area was quantified. Results were expressed as fold change normalized to the control group. The experiments were repeated 3 times $(n=12)$.

\subsection{Mitochondrial bioenergetics.}

2.7.1. Oxygen consumption rate (OCR).-For embryonic assays, each experiment consisted of 2 Petri dishes, while for larval assays each experiment consisted of 1 petri dish. Animals were assayed at $24 \mathrm{hpf}$ (embryos) or $96 \mathrm{hpf}$ (larvae). The in vivo OCR was assessed in $24 \mathrm{hpf}$ or $96 \mathrm{hpf}$ embryos using the XFe24 Extracellular Flux Analyzer (Agilent Instruments, Santa Clara, CA, USA) according to previously established protocols ${ }^{19}$, 20 .The experiments were repeated 3 times ( 96 hpf, $\mathrm{n}=15-18$ ) or 4 times ( $24 \mathrm{hpf}, \mathrm{n}=20-24$ ). Further details of the assay are discussed in the Supplementary Material.

2.7.2. NADH production.-Each experiment consisted of 2 Petri dishes per group, and 5 animals were randomly chosen per dish. Animals were assayed at $24 \mathrm{hpf}$ or $96 \mathrm{hpf}$ according to a previously published protocol using Alamar Blue (Thermo Fisher Scientific) to assess in vivo cumulative NADH production ${ }^{21}$.

Fluorescence was measured using a FLUOstar Optima at 530/10 nm (ex) and 590-10 nm (em) in cycles of $10 \mathrm{~min}$ over $24 \mathrm{~h}$, at $28^{\circ} \mathrm{C}$. The change on fluorescence values between the end and start of the assay was calculated, normalized to the control group and expressed as relative fluorescence change. The experiment was repeated 3 times $(n=30)$.

2.7.3 Mitochondrial content.-For this assay, $\operatorname{Tg}$ (MLS-EGFP) embryos were used for the exposures. This transgenic line expresses EGFP in mitochondria using an EGFP fused to the zebrafish COX VIII mitochondrial localization sequence (MLS). Each experiment consisted of 2 Petri dishes, and 5 embryos ( $24 \mathrm{hpf}$ ) or larvae ( $96 \mathrm{hpf}$ ) were randomly selected. PTU was used to prevent pigmentation, as described in section 2.5.

Animals were individually transferred to a 96-well zebrafish imaging plate (ZF plate, Hashimoto Electronic Industry, Takasucho, Japan) with the addition of $100 \mu \mathrm{L}$ tricaine (200 $\mathrm{mg} / \mathrm{ml}$ ). Images were acquired using a BZ-X700 automated fluorescence microscope (Keyence Corporation of America, Itasca, IL, USA) with 470/40 nm (em) and 525/50 nm (ex) filters. Fluorescence was quantified using ImageJ software and expressed as fold change normalized to the control group. The experiment was repeated 3 times $(n=30)$.

\subsection{Statistics}

The results are presented as a scatter plot and mean. All data were analyzed for normal distribution (Shapiro-Wilk and Kolgomorov-Smirnov tests) and transformed if necessary. Data were further analyzed by Kruskal-Wallis followed by Dunn's posthoc (for non-normal 
data) or two-way ANOVA followed by Tukey's posthoc. These analyses were conducted using Graph Pad Prism 6.0 (San Diego, CA, USA). For all endpoints, p-value $<0.05$ was considered statistically significant.

\section{Results and Discussion}

The individual toxicity of Nano-PS and ERSE was assessed to zebrafish embryonic and larval development up to $96 \mathrm{hpf}$. As shown in our previous studies with similar nanoplastic particles ${ }^{8}$, Nano-PS did not cause mortality at concentrations as high as $10 \mathrm{ppm}$ (Fig. S3). ERSE exposures up to 5\% (final dilution) also did not affect the zebrafish larval survival (Fig. S4), similarly to previous results from our group 22.

Analyses of developmental deformities were also conducted (Fig. 1). Nano-PS exposures did not induce visible deformities, but a trend of increased curved tail rates at $1 \mathrm{ppm}$ was detected (Fig. 1A, p $=0.077$ against $\mathrm{Ctl}$ group). On the other hand, ERSE exposures were teratogenic to the larvae, as the frequency rates for curved tail, jaw deformities, pericardial edema, and string heart all increased. The mean value for curved tails with 5\% ERSE was approximately $40 \%$ (Fig. 1B) and jaw deformities were detected in approximately $25 \%$ of the larvae at 2\% and 5\% ERSE (Fig. 1C). The rates for pericardial edema and string heart were much higher: approximately $40 \%$ and $83 \%$ of the larvae presented pericardial edema with 2\% and 5\% ERSE, respectively (Fig. 1D) and 68\% of them had string heart at 5\% ERSE (Fig. 1E). This profound effect of ERSE on cardiac deformities has already been demonstrated in embryos or larvae of zebrafish ${ }^{22}$ and killifish ${ }^{23,24}$

To characterize the interaction of Nano-PS on PAH toxicity, we used co-exposures of 10 ppm Nano-PS and $1 \%, 2 \%$ or $5 \%$ ERSE. This concentration of Nano-PS was shown to not induce mortality or deformities in zebrafish larvae; it was also the concentration previously used to characterize the uptake and toxicity of nanoplastics in zebrafish larvae ${ }^{8}$. These ERSE concentrations also offered a range of deformities from lesser (1\% ERSE) to greater (5\% ERSE) extent. None of the combined exposures caused significant mortality to zebrafish embryos and larvae (Fig. S5). Once more, ERSE exposures caused high rates of developmental deformities, but the co-exposure of ERSE with Nano-PS caused a partial protective effect (Fig. 2 A-E). Jaw deformities and pericardial edema rates decreased for $2 \%$ ERSE, while the frequencies for string heart and fin deformities (Fig. 2E) decreased for $5 \%$ ERSE. Despite these protective effects, Nano-PS co-exposure caused a significant $18 \%$ increase in the heartbeat rate at 5\% ERSE. Previous results had shown that Nano-PS embryonic or maternal exposure can cause the uptake of nanoplastics to the heart and decrease the heartbeat rate of zebrafish at 48 or $72 \mathrm{hpf}^{7,8}$. These results current indicated that Nano-PS seems to decrease overall PAH uptake during ERSE exposure and therefore decreases the expected teratogenicity. However, it can also increase the toxicity of sorbed chemicals in target organs such as the heart through other mechanisms.

Combined exposures of nanoplastics and other toxicants have shown different results regarding toxicity. A study investigating the interaction of pyrene and Nano-PS $(568 \mathrm{~nm})$ indicated that Nano-PS accumulated pyrene at levels approximately 488 times higher than the exposure medium (seawater) after 14 days of incubation. Clamworms Perinereis 
aibuhitensispyrene co-exposed to these chemicals had lower bioaccumulation levels of pyrene, possibly due to the sorption and removal of free pyrene by Nano-PS in the exposure medium, while Nano-PS accounted for approximately $1 \%$ of the total pyrene accumulated by the organisms ${ }^{25}$. On the other hand, copper ions were found to not adsorb to the surface of carboxylated Nano-PS (87 to $106 \mathrm{~nm}$ ) and to not alter the toxicity of these nanoplastics to the freshwater alga Raphidocelis subcapitata ${ }^{26}$. When compared to larger polystyrene particles $(0.5-15 \mu \mathrm{m}), 50 \mathrm{~nm}$ Nano-PS were more toxic to Daphnia magna, induced the sorption of phenanthrene to their surface, and increased the bioaccumulation and toxicity of phenanthrene ${ }^{27}$. It is thus important to better comprehend how plastics can interact with other contaminants and modulate their uptake and toxicity. Such effects can be difficult to predict, as they are dependent on the polymer type, size and charge of the plastic particles, type of co-contaminant, and their concentrations.

In this study, we hypothesized that the high surface to volume ratio of the plastic nanoparticles and the hydrophobic nature of Nano-PS and ERSE could drive the sorption of free PAHs to the Nano-PS, decreasing the concentration of bioavailable-PAHs $11,25,28$. To investigate such interaction, DLS and zeta potential were assayed in 10 ppm Nano-PS incubated with or without 5\% ERSE (Fig. S3). The results indicate that in the absence of ERSE, Nano-PS have relatively low aggregation rate and polydispersity index (0.08-0.32) after 96 hours of incubation. It also increases the average diameter from $44.73 \mathrm{~nm}$ to 129.9 $\mathrm{nm}$ and the zeta potential from $-38.0 \mathrm{mV}$ to $-8.39 \mathrm{mV}$ (possibly due to interactions with cations from the medium such as $\mathrm{Na}^{+}, \mathrm{K}^{+}, \mathrm{Ca}^{2+}$, and $\mathrm{Mg}^{+}$). The presence of ERSE leads to fast aggregation of Nano-PS, resulting in average diameters higher than $500 \mathrm{~nm}$, much higher polydispersity index (0.54-0.72), and a minor increase in the zeta potential (to -27.6 $\mathrm{mV}$ ). After 96 hours and in the absence of ERSE, 79\% of the Nano-PS mass was detected as particles with a size smaller than $100 \mathrm{~nm}$, while this value decreases to about $31 \%$ in the presence of ERSE. Sorption of PAHs to the surface of Nano-PS is expected to decrease the interaction with cations available in the exposure medium and to increase the lipophilicity of Nano-PS and, promoting further aggregation and sorption of PAHs. Uptake of 200 and 500 $\mathrm{nm}$ Nano-PS particles was shown to be lower than $50 \mathrm{~nm}$ Nano-PS particles in zebrafish embryos $^{29}$, suggesting that PAHs sorbed to agglomerates of Nano-PS may have limited uptake and bioaccumulation in early life zebrafish.

To further investigate this sorption effect, we estimated total PAH bioaccumulation in zebrafish larvae by fluorescence microscopy, taking advantage of the natural fluorescence of PAHs. The results indicated a clear increase in the overall green fluorescence at $96 \mathrm{hpf}$ for $2 \%$ and 5\% ERSE with or without Nano-PS (Fig. 3A). The analysis also detailed the fluorescence increase in areas such as yolk sac (Fig. 3B), head (Fig. 3C), heart (Fig. 3D), and tail (Fig. 3E). In the presence of Nano-PS, the fluorescence related to PAH accumulation by ERSE (5\%) decreased approximately 20 to $40 \%$ in the yolk sac, head, and heart. This was another indication that Nano-PS may be interacting with PAHs, promoting sorption and particle agglomeration, and decreasing their uptake, transport, and accumulation in specific organs. This could be one of the reasons for the lower teratogenicity detected at the Nano-PS + ERSE exposure groups. It is unclear if such sorption effect also alters the final composition of PAHs bioaccumulated in zebrafish larvae, what can play an important role in toxicity and remains to be investigated. Despite the lower PAH bioaccumulation in the heart 
at Nano-PS + 5\% ERSE 96 hpf larvae, this exposure group had increased heartbeat rate (Fig. 2F) at $48 \mathrm{hpf}$. This suggests that the high concentrations of PAHs in 5\% ERSE strongly induce cardiac deformities at later stages, but the interactive effects of Nano-PS and PAHs can induce changes on heartbeat rate independently from cardiac deformities.

Chemical characterizations of the ERSE mixture indicated the presence of strong aryl hydrocarbon receptor (AhR) agonist PAHs, such as 1,2-benzofluorene, 1,2-benzanthracene, benzo(k)fluoranthene and indeno(1,2,3-c,d)pyrene, as well as AhR antagonists such as dibenzothiophene and fluoranthene ${ }^{15}$. The increased EROD activity (Fig. 3F) detected at 96 hpf in larvae exposed to ERSE indicates that enough PAHs are accumulated in the organism to induce this sensitive molecular biomarker of exposure, despite the lower bioaccumulation PAHs mediated by Nano-PS co-exposure.

One of the known effects of PAHs in zebrafish early development is the disruption of the cardiovascular system ${ }^{18,30,31}$. By using Tg (Flk1:EGFP) zebrafish we analyzed the blood vessel formation in $96 \mathrm{hpf}$ larvae (Fig. 4). The head of the larvae was analyzed in detail, as it was a well-vascularized area shown to bioaccumulate PAHs in the presence of Nano-PS. Results indicated that 5\% ERSE decreased vascularization, but not in the presence of NanoPS (Fig. 4). The effects of ERSE on cardiovascular development were not surprising, as adverse effects on heart development were already detected (Figs 1 and 2). Additionally, some PAHs can cause up-or down-regulation of genes related to vascular development and circulatory function in zebrafish ${ }^{32}$.

Mitochondria can also be a target for PAH toxicity, as its high lipid content facilitates PAH uptake $^{33}$. In fact, previous data from our lab indicated that PAHs such as benzo(a)pyrene, phenanthrene, fluoranthene ${ }^{19}$ or complex PAHs mixtures ${ }^{24,34}$ can impair mitochondrial bioenergetics in embryonic or larval fish. As hydrophobic compounds with a small size, nanoplastics could also accumulate in the mitochondrial lipid environment and interfere with mitochondrial function and integrity. To investigate the bioenergetic impact of Nano-PS, ERSE, or both on developing zebrafish, we used a treatment of 2\% ERSE as higher concentrations caused pronounced developmental effects that could confound the results. There were three different approaches to investigate mitochondrial bioenergetics: (i) mitochondrial respiration by a set of in vivo OCR measurements, (ii) in vivo cumulative NADH production by the citric acid cycle, and (iii) in vivo mitochondrial content using $\mathrm{Tg}$ (MLS-EGFP) zebrafish.

At 24 hpf, embryos exhibited normal total basal respiration, including basal mitochondrial (Fig. 5B) and non-mitochondrial OCRs (Fig. 5). Total maximal and maximal mitochondrial respiration rates, as well as mitochondrial spare capacity, were also unaltered by all treatment conditions (Fig. 5). These same analyses were conducted at $96 \mathrm{hpf}$, and no effects were detected as well (Fig. S6).

Additional experiments indicated that the despite the normal basal OCR values, the fraction related to ATP production (ATP-linked OCR) decreased $49-65 \%$ in embryos exposed to Nano-PS with or without ERSE (Fig. 5). We also detected higher OCRs related to proton leak ( 6.5 fold) in these same exposure groups, leading to $50-60 \%$ decreased mitochondrial 
coupling efficiency (Fig. 5). Additional experiments excluded the possible intracellular interaction of Nano-PS with oligomycin A during the assay, preventing the expected inhibition of ATP synthase which would result in a false lower ATP-linked OCR (Fig. S8). This set of data was the first indicator of a Nano-PS-exclusive marker of toxicity and suggested that mitochondria can be a target organelle for Nano-PS. Analyses at $96 \mathrm{hpf}$ for ATP-linked and proton leak could not be conducted as the larvae are much less responsive to oligomycin A at our experimental conditions ${ }^{19}$.

Mitochondrial ATP production relies on a constant flow of NADH to the electron transfer chain (ETC). Alamar blue measures the NADH production efficiency of the citric acid cycle in embryos and larvae over time (Fig. 6). In $24 \mathrm{hpf}$ embryos, results indicate an increased production rate after exposure to Nano-PS $(\mathrm{p}=0.10)$ and a significant increase after the Nano-PS-ERSE co-exposure. At 96 hpf larvae, both groups displayed significantly higher NADH production rates. As the ATP-linked OCR was greatly affected by nanoplastic exposure, it is possible that this increase in NADH production was a compensatory mechanism to fuel the mitochondrial ETC with extra reducing equivalents for ATP production. Another possible scenario was that mitochondrial dysfunction caused by NanoPS slowed down NADH consumption by the ETC, resulting in an accumulation of NADH over time. Our data suggest a strong effect of Nano-PS on two important mitochondrial functions (NADH and ATP synthesis), but it remains unclear how Nano-PS are causing these effects.

Lastly, we estimated mitochondrial mass using the Tg (MLS-EGFP) zebrafish. An increase in embryonic total mitochondrial mass was detected in Nano-PS + ERSE embryos compared to Nano-PS embryos (Fig. S9). At $96 \mathrm{hpf}$, an increase in liver mitochondrial mass was detected with larvae exposed to ERSE only. These extra sets of data indicate that a compensatory mechanism may take place early on development when animals are exposed to two mitotoxicants such as NanoPS and PAHs. But this effect was transitory, as mitochondrial mass was not affected by Nano-PS at $96 \mathrm{hpf}$. As the main organ for PAH metabolism, the liver may require extra energy for PAH biotransformation, leading to increased mitochondrial biogenesis as detected in $96 \mathrm{hpf}$ larvae. Additional experiments regarding mitochondrial dynamics are still necessary to support such results and could be of great value to better understand the effects of nanoplastics at the mitochondrial level.

Previous studies from our laboratory investigated the mitochondrial bioenergetics of zebrafish embryos exposed to Nano-PS through parental or waterborne exposure, but no signs of mitochondrial disruption were detected ${ }^{7,8}$. However, the ATP-linked OCR proton leak OCR, and the mitochondrial coupling efficiency were not analyzed in those studies. Interestingly, PVC microparticles increased the accumulation of an antidepressant drug in mitochondria of the fish M. anguillicaudatus, possibly due to the sorption and transport of co-contaminants to the mitochondria ${ }^{35}$. Mussels (Mytilus edulis) exposed to polystyrene microplastics $(10-90 \mu \mathrm{m})$ had higher energy consumption and activity of the ETC, another indication that the plastic particles could be transported to mitochondria or affect their bioenergetics ${ }^{36}$. While plastics at the micrometer size would exceed the size limitation for mitochondrial uptake, nanoplastics may not, as their size can be up to 3 orders of magnitude smaller than an individual mitochondrion. 
There are very few studies indicating the cellular targets of nanoplastics. Nano-PS can interact with and disrupt the plasma membrane, lysosomes, cytoskeleton (actin), and metabolism-related proteins (CYP450 enzymes) ${ }^{37}$ In vitro cell studies showed mitochondrial uptake of nanoplastics, which was associated with increased mitochondrial damage and oxidative stress ${ }^{38,39}$. It is known that mitochondrial uptake and disruption is a major toxicity pathway for other type of nanoparticles, such as zinc oxide and graphene oxide metallic ones 40,41 . A recent study also identified vacuoles partial disruption of the cristae in the mitochondria of $48 \mathrm{hpf}$ zebrafish embryos, what was correlated with increased mitochondrial ROS production ${ }^{29}$. Although these effects were not strong enough to induce cellular death and mortality of zebrafish embryos, such exposure increased the mitochondrial and developmental toxicity of gold ions ${ }^{29}$. Our data also point to mitochondrial uptake of Nano-PS and subsequent disruption, but further studies are necessary to characterize this process and confirm it as a molecular initiation event for further adverse outcomes, especially in the presence of multiple stressors.

Our results indicate that Nano-PS promoted the sorption of PAHs from the exposure medium, increasing the agglomeration rate of Nano-PS and decreasing the bioavailability and bioaccumulation of PAHs. Although chemical sorption is a characteristic shared by many plastics particles, the higher surface area and the expected higher ingestion and biodistribution rates of nanoplastics have the potential to exacerbate their threat as pollutant carriers. In the presence of complex mixtures, Nano-PS can interact with each pollutant at different levels, potentially changing the chemical composition of the free and sorbed pollutants and the resulting toxicity of the exposure medium. While this study focused on these interactions in a mixed exposure scenario, future studies pre-loading Nano-PS with complex mixtures will help to understand how the sorption rates of each individual chemical can contribute to predicting the potential toxicity of Nano-PS, how chemically stable these chemicals are once sorbed to Nano-PS, and if Nano-PS can alter the target of the sorbed chemicals after ingestion. Lastly, while the use of commercial polystyrene nanoparticles with homogenous spherical shape may not truly represent the nanoplastics found in the environment, our results indicate the potential risk of nano-polymers to cause mitochondrial energy disruption, the decline in energy efficiency, and the differential mitochondrial uptake of associated contaminants. More studies are still necessary to address such effects with environmental-relevant nano-polymers and under energy-demanding scenarios, which could help to address the environmental threats of nanoplastic pollution.

\section{Supplementary Material}

Refer to Web version on PubMed Central for supplementary material.

\section{Acknowledgments}

We thank all the members of the Di Giulio laboratory for help with zebrafish husbandry. We would also like to thank Casey Lindberg for her help with the manuscript revision. The research was supported by Duke's Superfund Research Center (NIEHS P42-ES010356) and National Science Foundation (NSF) and the Environmental Protection Agency (EPA) under NSF Cooperative Agreement EF-0830093 and DBI-1266252, Center for the Environmental Implications of Nanotechnology (CEINT). 


\section{References}

1. Avio CG; Gorbi S; Regoli F, Plastics and microplastics in the oceans: From emerging pollutants to emerged threat. Mar Environ Res 2017, 128, 2-11. [PubMed: 27233985]

2. Gigault J; Halle AT; Baudrimont M; Pascal PY; Gauffre F; Phi TL; El Hadri H; Grassl B; Reynaud S, Current opinion: What is a nanoplastic? Environ Pollut 2018, 235, 1030-1034. [PubMed: 29370948]

3. Ivleva NP; Wiesheu AC; Niessner R, Microplastic in Aquatic Ecosystems. Angew Chem Int Ed Engl 2017, 56, (7), 1720-1739. [PubMed: 27618688]

4. Ward JE; Kach DJ, Marine aggregates facilitate ingestion of nanoparticles by suspension-feeding bivalves. Mar Environ Res 2009, 68, (3), 137-42. [PubMed: 19525006]

5. Chae Y; Kim D; Kim SW; An YJ, Trophic transfer and individual impact of nano-sized polystyrene in a four-species freshwater food chain. Sci Rep 2018, 8, (1), 284. [PubMed: 29321604]

6. Brun NR; Beenakker MMT; Hunting ER; Ebert D; Vijver MG, Brood pouch-mediated polystyrene nanoparticle uptake during Daphnia magna embryogenesis. Nanotoxicology 2017, 11, (8), 10591069. [PubMed: 29083253]

7. Pitt JA; Trevisan R; Massarsky A; Kozal JS; Levin ED; Di Giulio RT, Maternal transfer of nanoplastics to offspring in zebrafish (Danio rerio): A case study with nanopolystyrene. Sci Total Environ 2018, 643, 324-334. [PubMed: 29940444]

8. Pitt JA; Kozal JS; Jayasundara N; Massarsky A; Trevisan R; Geitner N; Wiesner M; Levin ED; Di Giulio RT, Uptake, tissue distribution, and toxicity of polystyrene nanoparticles in developing zebrafish (Danio rerio). Aquat Toxicol 2018, 194, 185-194. [PubMed: 29197232]

9. Liu Z; Cai M; Yu P; Chen M; Wu D; Zhang M; Zhao Y, Age-dependent survival, stress defense, and AMPK in Daphnia pulex after short-term exposure to a polystyrene nanoplastic. Aquat Toxicol 2018, 204, 1-8. [PubMed: 30153596]

10. Veneman WJ; Spaink HP; Brun NR; Bosker T; Vijver MG, Pathway analysis of systemic transcriptome responses to injected polystyrene particles in zebrafish larvae. Aquat Toxicol 2017, 190, 112-120. [PubMed: 28704660]

11. Liu LJ; Fokkink R; Koelmans AA, Sorption of polycyclic aromatic hydrocarbons to polystyrene nanoplastic. Environmental Toxicology and Chemistry 2016, 35, (7), 1650-1655. [PubMed: 26588181]

12. Wright SL; Thompson RC; Galloway TS, The physical impacts of microplastics on marine organisms: a review. Environ Pollut 2013, 178, 483-92. [PubMed: 23545014]

13. Rochman CM; Browne MA; Halpern BS; Hentschel BT; Hoh E; Karapanagioti HK; Rios-Mendoza LM; Takada H; Teh S; Thompson RC, Classify plastic waste as hazardous. Nature 2013, 494, (7436), 169-171. [PubMed: 23407523]

14. Nasevicius A; Ekker SC, Effective targeted gene 'knockdown' in zebrafish. Nat Genet 2000, 26, (2), 216-220. [PubMed: 11017081]

15. Fang M; Getzinger GJ; Cooper EM; Clark BW; Garner LV; Di Giulio RT; Ferguson PL; Stapleton HM, Effect-directed analysis of Elizabeth River porewater: developmental toxicity in zebrafish (Danio rerio). Environ Toxicol Chem 2014, 33, (12), 2767-74. [PubMed: 25196082]

16. Truong L; Bugel SM; Chlebowski A; Usenko CY; Simonich MT; Simonich SLM; Tanguay RL, Optimizing multi-dimensional high throughput screening using zebrafish. Reprod Toxicol 2016, 65, 139-147. [PubMed: 27453428]

17. Noury P; Geffard O; Tutundjian R; Garric J, Non destructive in vivo measurement of ethoxyresorufin biotransformation by zebrafish prolarva: development and application. Environ Toxicol 2006, 21, (4), 324-31. [PubMed: 16841310]

18. Massarsky A; Prasad GL; Di Giulio RT, Total particulate matter from cigarette smoke disrupts vascular development in zebrafish brain (Danio rerio). Toxicol Appl Pharmacol 2018, 339, 85-96. [PubMed: 29221954]

19. Raftery TD; Jayasundara N; Di Giulio RT, A bioenergetics assay for studying the effects of environmental stressors on mitochondrial function in vivo in zebrafish larvae. Comp Biochem Physiol C Toxicol Pharmacol 2017, 192, 23-32. [PubMed: 27939721] 
20. Stackley KD; Beeson CC; Rahn JJ; Chan SS, Bioenergetic profiling of zebrafish embryonic development. PLoS One 2011, 6, (9), e25652.

21. Williams SY; Renquist BJ, High Throughput Danio Rerio Energy Expenditure Assay. J Vis Exp 2016, (107), e53297.

22. Fleming CR; Di Giulio RT, The role of CYP1A inhibition in the embryotoxic interactions between hypoxia and polycyclic aromatic hydrocarbons (PAHs) and PAH mixtures in zebrafish (Danio rerio). Ecotoxicology 2011, 20, (6), 1300-14. [PubMed: 21706407]

23. Brown DR; Bailey JM; Oliveri AN; Levin ED; Di Giulio RT, Developmental exposure to a complex PAH mixture causes persistent behavioral effects in naive Fundulus heteroclitus (killifish) but not in a population of PAH-adapted killifish. Neurotoxicol Teratol 2016, 53, 55-63. [PubMed: 26548404]

24. Lindberg CD; Jayasundara N; Kozal JS; Leuthner TC; Di Giulio RT, Resistance to polycyclic aromatic hydrocarbon toxicity and associated bioenergetic consequences in a population of Fundulus heteroclitus. Ecotoxicology 2017, 26, (3), 435-448. [PubMed: 28213827]

25. Jiang XT; Tian LL; Ma YN; Ji R, Quantifying the bioaccumulation of nanoplastics and PAHs in the clamworm Perinereis aibuhitensis. Science of the Total Environment 2019, 655, 591-597. [PubMed: 30476839]

26. Bellingeri A; Bergami E; Grassi G; Faleri C; Redondo-Hasselerharm P; Koelmans AA; Corsi I, Combined effects of nanoplastics and copper on the freshwater alga Raphidocelis subcapitata. Aquatic Toxicology 2019, 210, 179-187. [PubMed: 30870664]

27. Ma YN; Huang AN; Cao SQ; Sun FF; Wang LH; Guo HY; Ji R, Effects of nanoplastics and microplastics on toxicity, bioaccumulation, and environmental fate of phenanthrene in fresh water. Environmental Pollution 2016, 219, 166-173. [PubMed: 27814532]

28. Lin W; Jiang RF; Xiong YX; Wu JY; Xu JQ; Zheng J; Zhu F; Ouyang GF, Quantification of the combined toxic effect of polychlorinated biphenyls and nano-sized polystyrene on Daphnia magna. Journal of Hazardous Materials 2019, 364, 531-536. [PubMed: 30388636]

29. Lee WS; Cho HJ; Kim E; Huh YH; Kim HJ; Kim B; Kang T; Lee JS; Jeong J, Bioaccumulation of polystyrene nanoplastics and their effect on the toxicity of Au ions in zebrafish embryos (vol 11, pg 3173, 2019). Nanoscale 2019, 11, (7), 3396-3396. [PubMed: 30608099]

30. Elie MR; Choi J; Nkrumah-Elie YM; Gonnerman GD; Stevens JF; Tanguay RL, Metabolomic analysis to define and compare the effects of PAHs and oxygenated PAHs in developing zebrafish. Environ Res 2015, 140, 502-510. [PubMed: 26001975]

31. Incardona JP; Collier TK; Scholz NL, Defects in cardiac function precede morphological abnormalities in fish embryos exposed to polycyclic aromatic hydrocarbons. Toxicol Appl Pharmacol 2004, 196, (2), 191-205. [PubMed: 15081266]

32. Goodale BC; Tilton SC; Corvi MM; Wilson GR; Janszen DB; Anderson KA; Waters KM; Tanguay $\mathrm{RL}$, Structurally distinct polycyclic aromatic hydrocarbons induce differential transcriptional responses in developing zebrafish. Toxicol Appl Pharm 2013, 272, (3), 656-670.

33. Meyer JN; Leung MCK; Rooney JP; Sendoel A; Hengartner MO; Kisby GE; Bess AS, Mitochondria as a Target of Environmental Toxicants. Toxicological Sciences 2013, 134, (1), 117. [PubMed: 23629515]

34. Massarsky A; Jayasundara N; Bailey JM; Oliveri AN; Levin ED; Prasad GL; Di Giulio RT, Teratogenic, bioenergetic, and behavioral effects of exposure to total particulate matter on early development of zebrafish (Danio rerio) are not mimicked by nicotine. Neurotoxicology and Teratology 2015, 51, 77-88. [PubMed: 26391568]

35. Qu H; Ma R; Wang B; Yang J; Duan L; Yu G, Enantiospecific toxicity, distribution and bioaccumulation of chiral antidepressant venlafaxine and its metabolite in loach (Misgurnus anguillicaudatus) co-exposed to microplastic and the drugs. J Hazard Mater 2018.

36. Van Cauwenberghe L; Claessens M; Vandegehuchte MB; Janssen CR, Microplastics are taken up by mussels (Mytilus edulis) and lugworms (Arenicola marina) living in natural habitats. Environ Pollut 2015, 199, 10-7. [PubMed: 25617854]

37. Frohlich E, Cellular targets and mechanisms in the cytotoxic action of non-biodegradable engineered nanoparticles. Curr Drug Metab 2013, 14, (9), 976-88. [PubMed: 24160294] 
38. Ruenraroengsak P; Tetley TD, Differential bioreactivity of neutral, cationic and anionic polystyrene nanoparticles with cells from the human alveolar compartment: robust response of alveolar type 1 epithelial cells. Part Fibre Toxicol 2015, 12, 19. [PubMed: 26133975]

39. Xia T; Kovochich M; Brant J; Hotze M; Sempf J; Oberley T; Sioutas C; Yeh JI; Wiesner MR; Nel $\mathrm{AE}$, Comparison of the abilities of ambient and manufactured nanoparticles to induce cellular toxicity according to an oxidative stress paradigm. Nano Lett 2006, 6, (8), 1794-807. [PubMed: 16895376]

40. Chen Y; Hu X; Sun J; Zhou Q, Specific nanotoxicity of graphene oxide during zebrafish embryogenesis. Nanotoxicology 2016, 10, (1), 42-52. [PubMed: 25704117]

41. Trevisan R; Delapedra G; Mello DF; Arl M; Schmidt EC; Meder F; Monopoli M; Cargnin-Ferreira E; Bouzon ZL; Fisher AS; Sheehan D; Dafre AL, Gills are an initial target of zinc oxide nanoparticles in oysters Crassostrea gigas, leading to mitochondrial disruption and oxidative stress. Aquat Toxicol 2014, 153, 27-38. [PubMed: 24745718] 

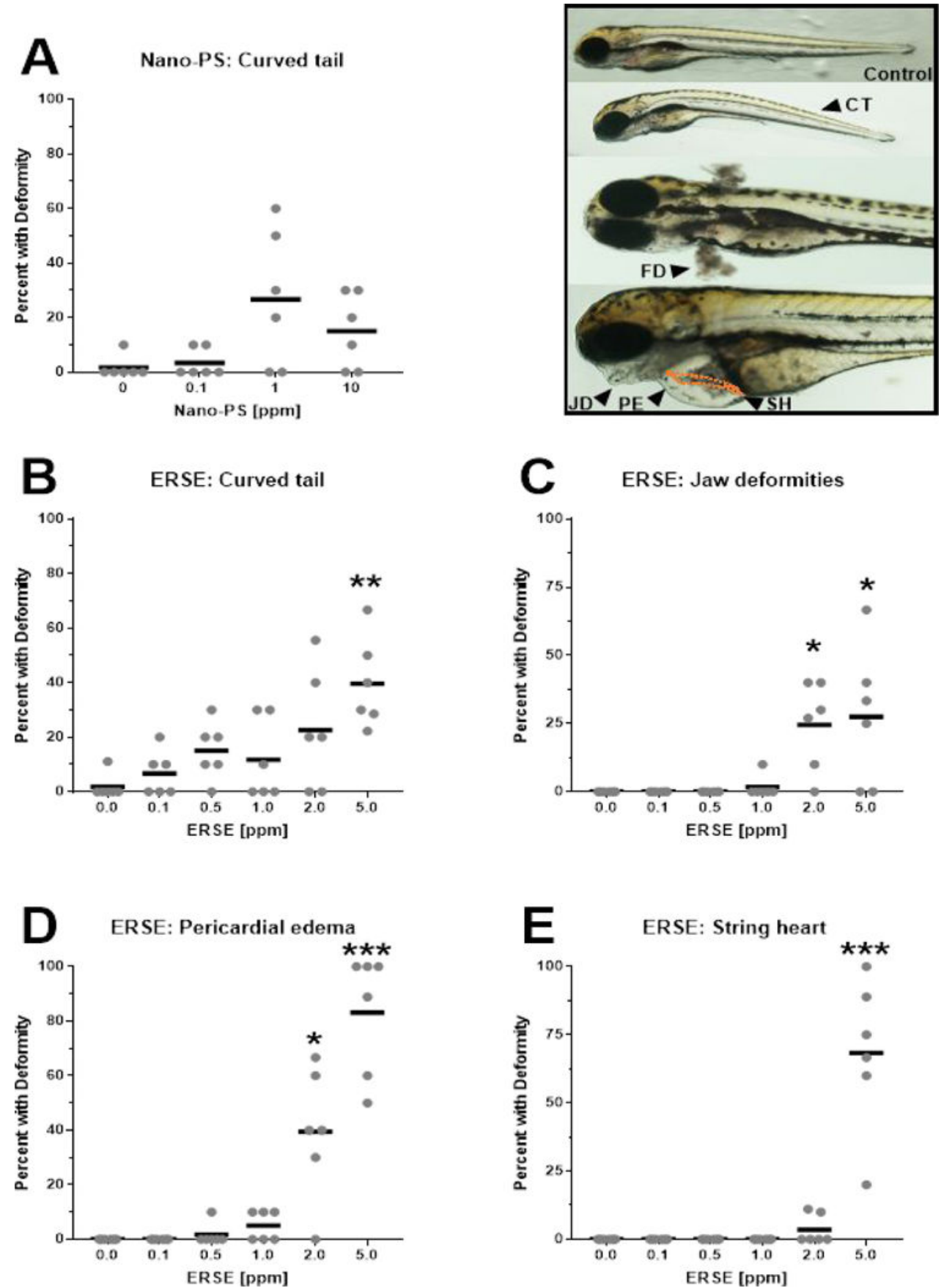

Figure 1:

Deformities rates of zebrafish larvae exposed to $44 \mathrm{~nm}$ nanopolystyrene particles (Nano-PS) (A) or a real-world environmental PAH mixture (Elizabeth River Sediment Extract - ERSE) (B -E). Animals were exposed at 6 hours post fertilization (hpf) to the indicated Nano-PS and ERSE concentrations, and the deformities were assessed at $96 \mathrm{hpf}$. The top right image panel shows representative images of the detected deformities: curved tail (CT), fin (FD) and jaw (JD) deformities, pericardial edema (PE) and string heart ( $\mathrm{SH}$, highlighted in orange dashed line). Results are presented as scatter plot $(n=6)$ and mean (black bar). Data were 
analyzed by Kruskal-Wallis followed by Dunn's posthoc, and differences against control are shown as $*(\mathrm{p}<0.05), * *(\mathrm{p}<0.01)$ or $* * *(\mathrm{p}<0.001)$. 
A
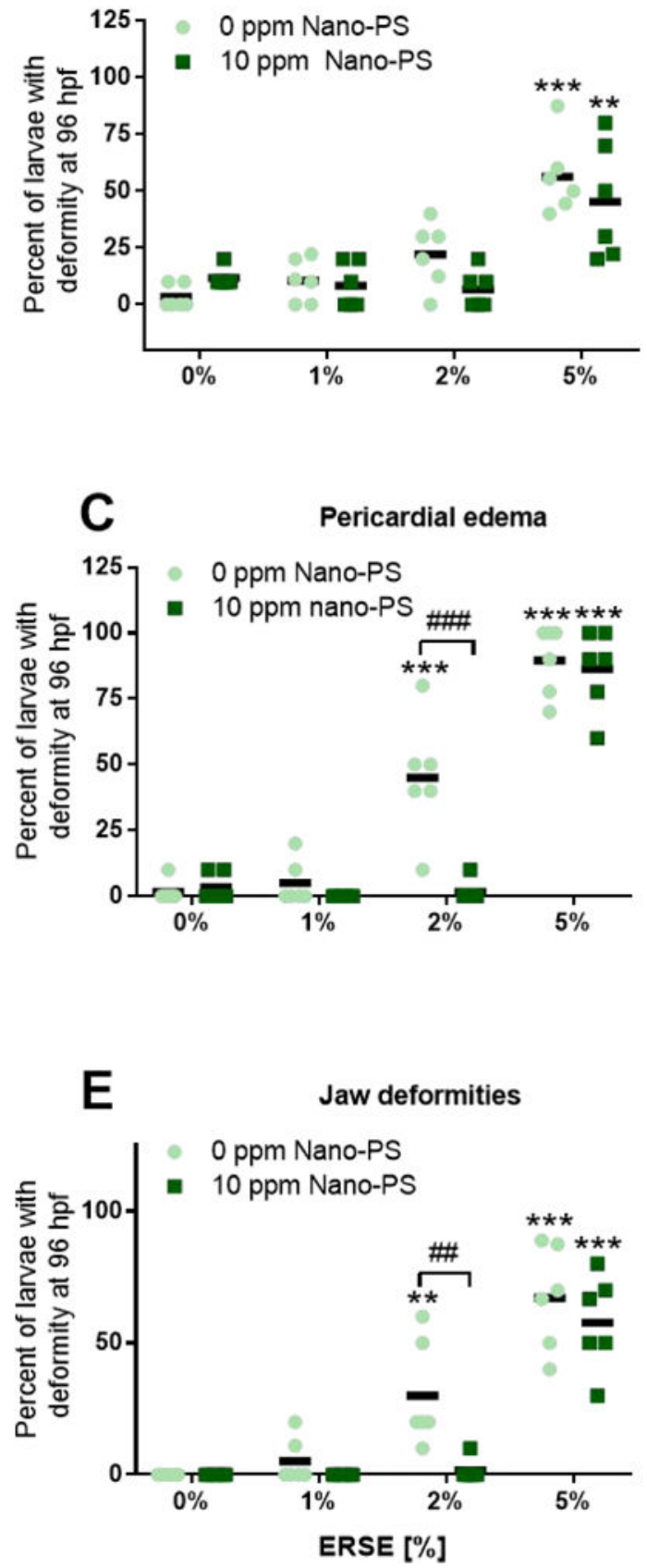

B

Fin deformities
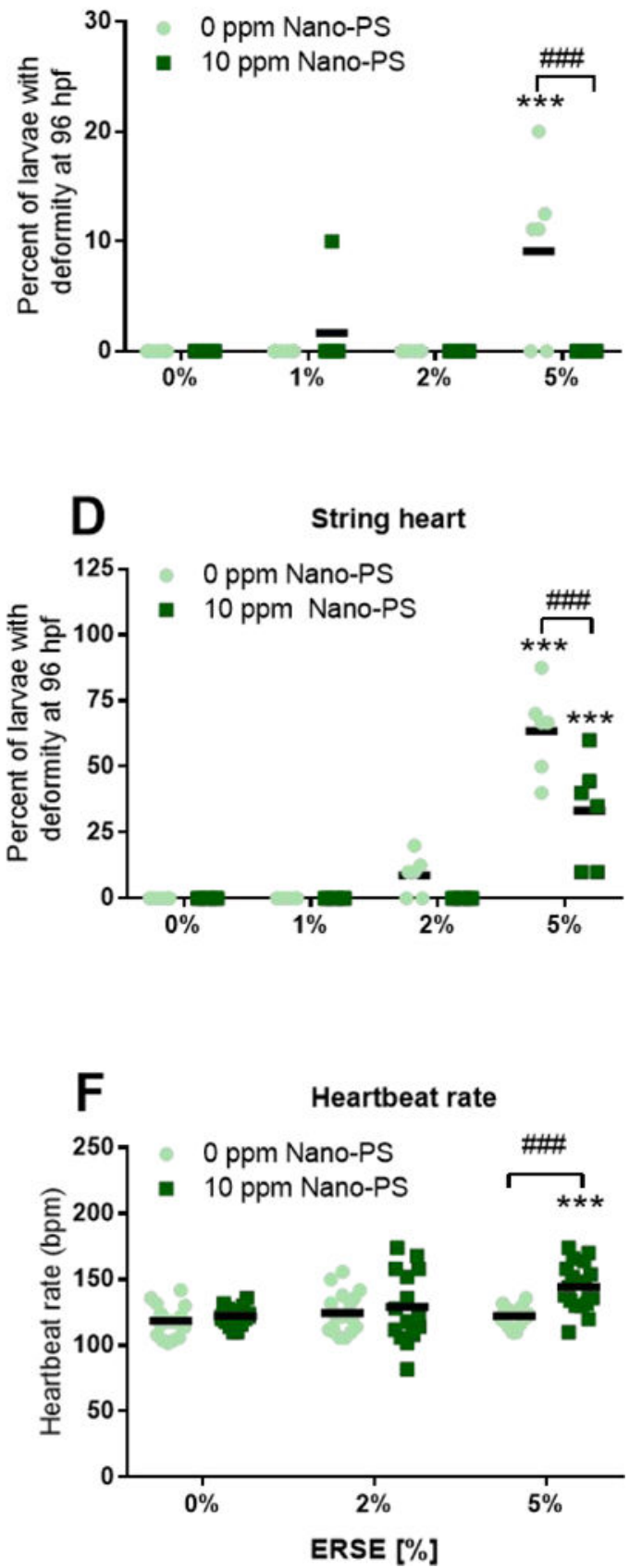

Figure 2:

Deformities and heartbeat rates of zebrafish larvae exposed to $44 \mathrm{~nm}$ nanopolystyrene particles (Nano-PS) in the presence of a real world environmental PAH mixture (Elizabeth River Sediment Extract - ERSE). Animals were co-exposed at 6 hours post fertilization (hpf) to $10 \mathrm{ppm}$ Nano-PS and $1 \%, 2 \%$ or $5 \%$ ERSE. Deformities were assessed at $96 \mathrm{hpf}$ (A $-\mathrm{E}$ ) and the heartbeat rate at $48 \mathrm{hpf}(\mathrm{F})$. Results are presented as scatter plot ( $\mathrm{n}=6$ for deformities, $\mathrm{n}=15-20$ for heartbeat) and mean (black bar). Data were analyzed by twoway ANOVA followed by Tukey's posthoc. Differences against the respective control group 
are shown as $* *(\mathrm{p}<0.01)$ and $* * *(\mathrm{p}<0.001)$, while differences between ERSE and ERSE + Nano-PS exposures are shown as \#\# $(\mathrm{p}<0.01)$ and \#\#\# $(\mathrm{p}<0.001)$. 

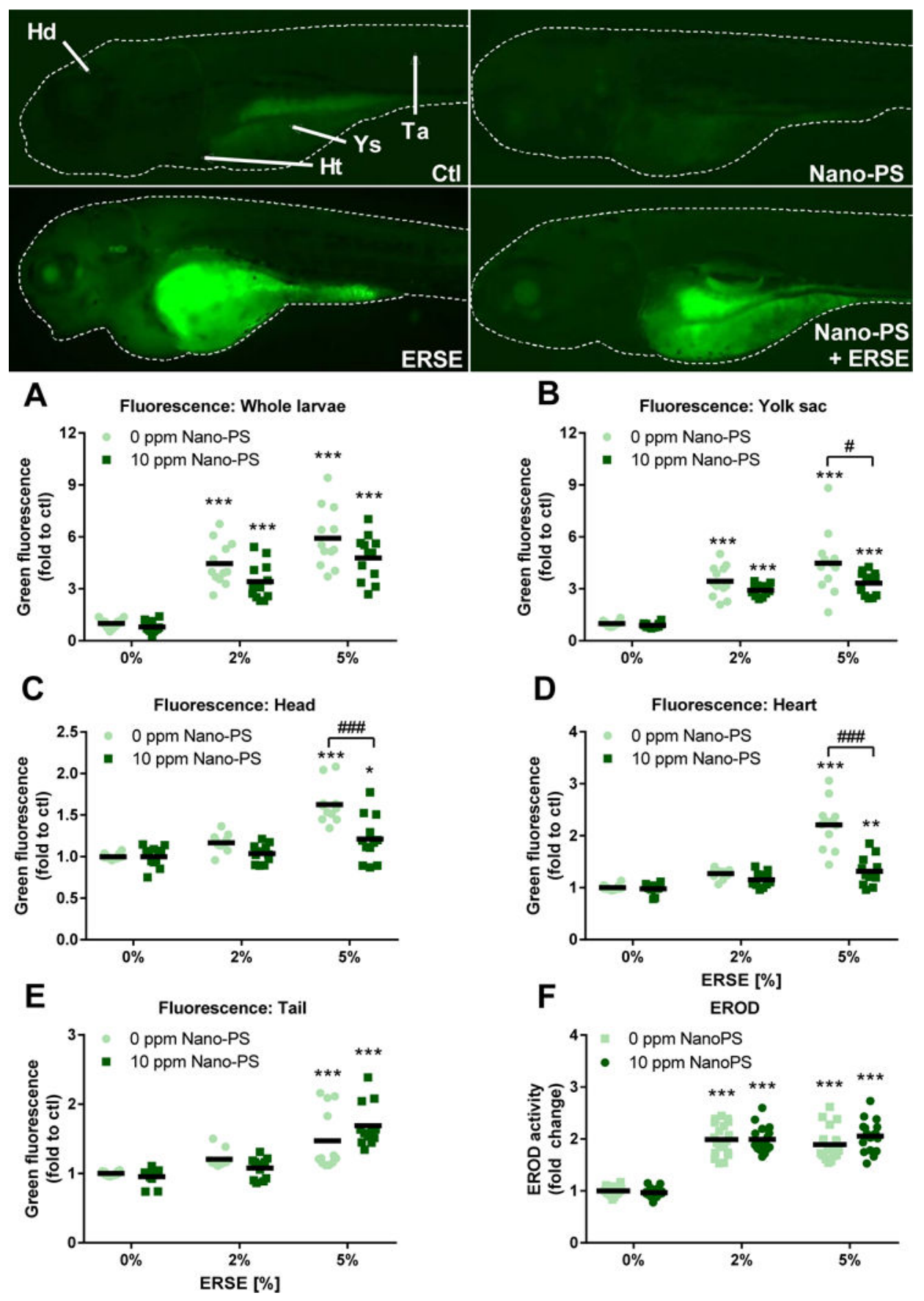

Figure 3:

EROD activity and total PAH bioaccumulation in zebrafish larvae exposed to $44 \mathrm{~nm}$ nanopolystyrene particles (Nano-PS) in the presence of a real world environmental PAH mixture (Elizabeth River Sediment Extract - ERSE). Animals were co-exposed at 6 hours post fertilization (hpf) to $10 \mathrm{ppm}$ Nano-PS and $2 \%$ or 5\% ERSE. The bioaccumulation was estimated by fluorescence microscopy at $96 \mathrm{hpf}$ in the whole larvae (A) or the indicated areas (B - E), and EROD activity was assessed in vivo at 96 hpf. Representative fluorescence microscopy images are shown at the top panel and indicate the analyzed areas: 
whole larvae (dashed line), head (Hd), heart (Ht), yolk sac (Ys), and tail (Ta). Results are presented as scatter plot $(\mathrm{n}=19-20$ for EROD activity, $\mathrm{n}=12$ for fluorescence microscopy) and mean (black bar). Data were analyzed by two-way ANOVA followed by Tukey's posthoc. Differences against the respective control group are shown as $*(\mathrm{p}<0.05)$ and $* * *(\mathrm{p}<0.001)$, while differences between ERSE and ERSE + Nano-PS exposures are shown as \# $(\mathrm{p}<0.05)$ and \#\# $(\mathrm{p}<0.001)$. 

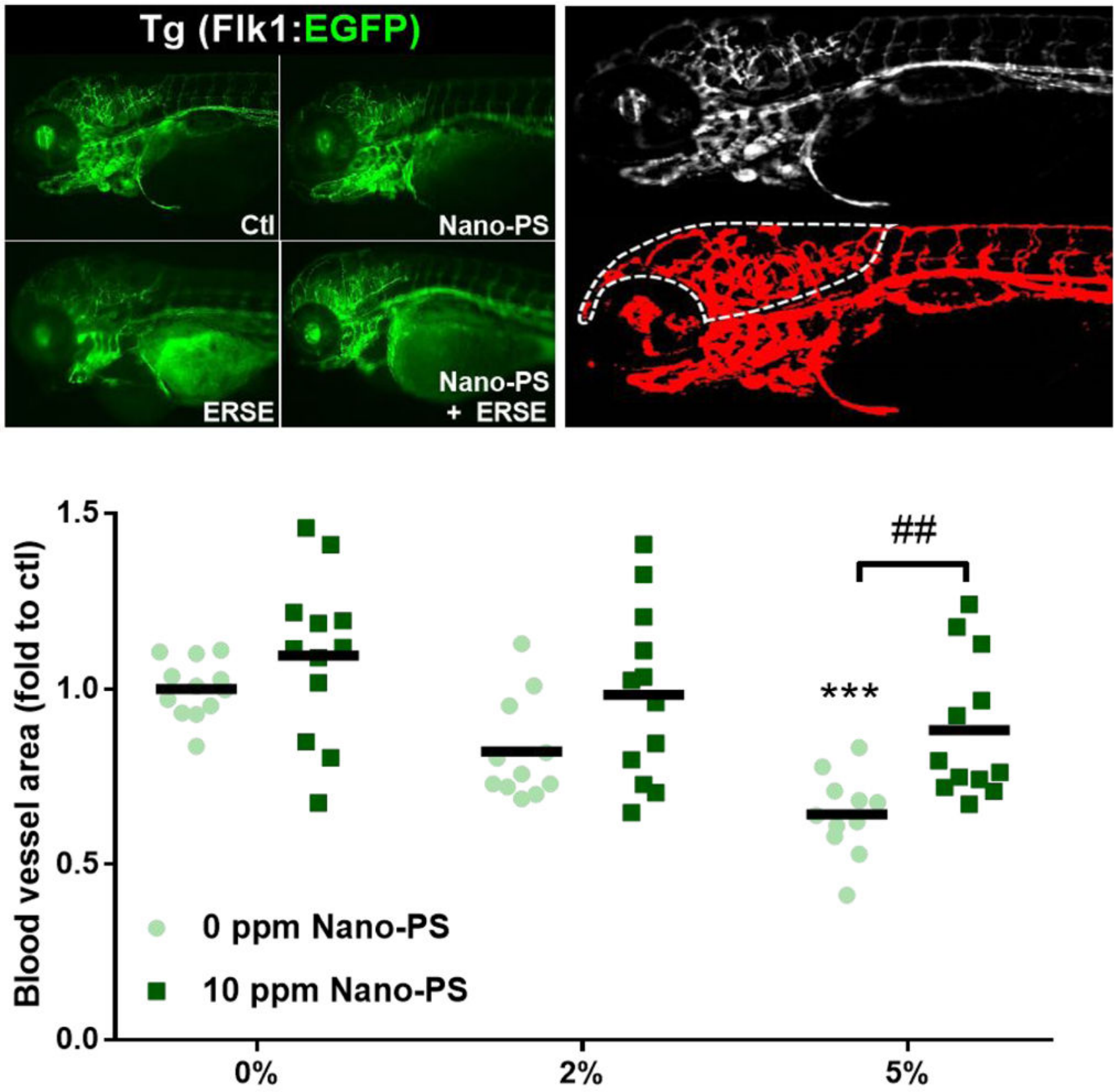

ERSE [\%]

Figure 4:

Blood vessel formation in zebrafish larvae exposed to $44 \mathrm{~nm}$ nanopolystyrene particles (Nano-PS) in the presence of a real world environmental PAH mixture (Elizabeth River Sediment Extract - ERSE). Animals were co-exposed at 6 hours post fertilization (hpf) to 10 ppm Nano-PS and $2 \%$ or 5\% ERSE. Blood vessels were assessed in vivo at 96 hpf by fluorescence microscopy using the transgenic line Tg (Flk1:EGFP), as shown in the top left panel. Further image analysis identified the blood vessels (red, top right panel) and quantified total blood vessel area in the head (dashed line, top right panel). Results are 
presented as scatter plot $(\mathrm{n}=11-12)$ and mean (black bar). Data were analyzed by two-way ANOVA followed by Tukey's posthoc. Differences against the respective control group are shown as $* * *(\mathrm{p}<0.001)$, while differences between ERSE and ERSE + Nano-PS exposures are shown as \#\# $(\mathrm{p}<0.01)$. 

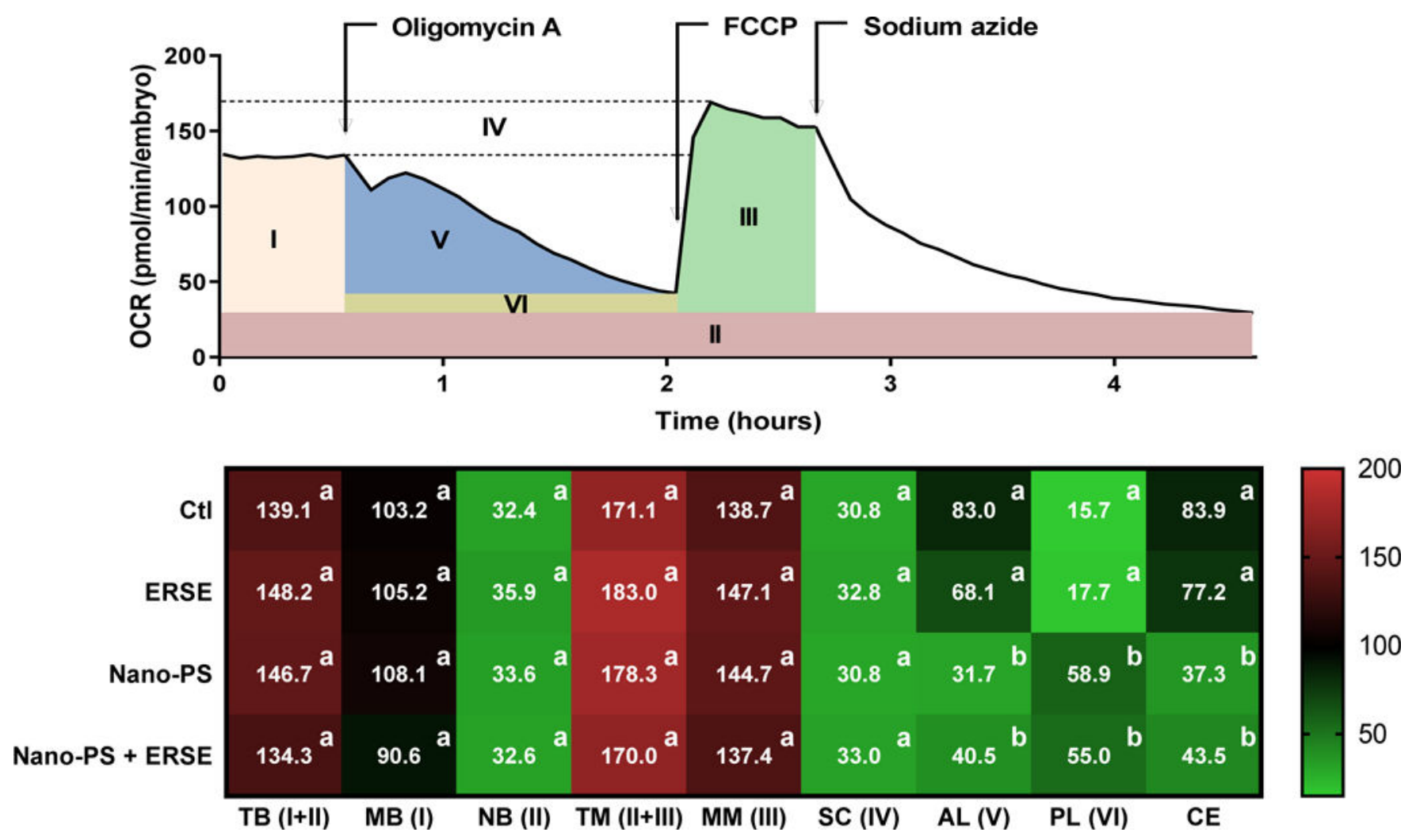

Figure 5:

Mitochondrial bioenergetics in zebrafish embryos exposed to $44 \mathrm{~nm}$ nanopolystyrene particles (Nano-PS) in the presence of a real world environmental PAH mixture (Elizabeth River Sediment Extract - ERSE). Animals were co-exposed at 6 hours post fertilization (hpf) to $10 \mathrm{ppm}$ Nano-PS and/or 2\% ERSE. Oxygen consumption rate (OCR) was assessed in vivo at $24 \mathrm{hpf}$. The top panel depicts the use of chemical agents to increase mitochondrial OCR to its maximal (FCCP), to completely block it (sodium azide), or to block only the mitochondrial ATP-linked OCR (oligomycin A), and the subsequent division of organismal bioenergetics into different categories (I - VI). Further details are provided in Fig. S1 and the OCR values overtime are shown in Fig. S7. The heatmap below shows the mean values ( $n=20-23$ ) for each bioenergetics fraction (with Roman numerals indicating the corresponding fraction in the top panel) as follow: TB (total basal), MB (mitochondrial basal), NB (non-mitochondrial basal), TM (total maximal), MM (mitochondrial maximal), SC (mitochondrial spare capacity), AL (ATP-linked) and PL (proton leak), while CE indicates the estimated mitochondrial coupling efficiency. Data were analyzed by two-way ANOVA followed by Tukey's posthoc. Groups with different letters are significantly different from each other $(\mathrm{p}<0.001)$. All parameters are shown as OCR ( $\mathrm{pmol} / \mathrm{min} /$ embryo), except for $\mathrm{CE}$ which is shown as percentage. Please note that the top panel indicates the use of oligomycin A, FCCP and sodium azide in a single experiment, but oligomycin A and FCCP were used in separate experiments to avoid possible interference on OCR values. 


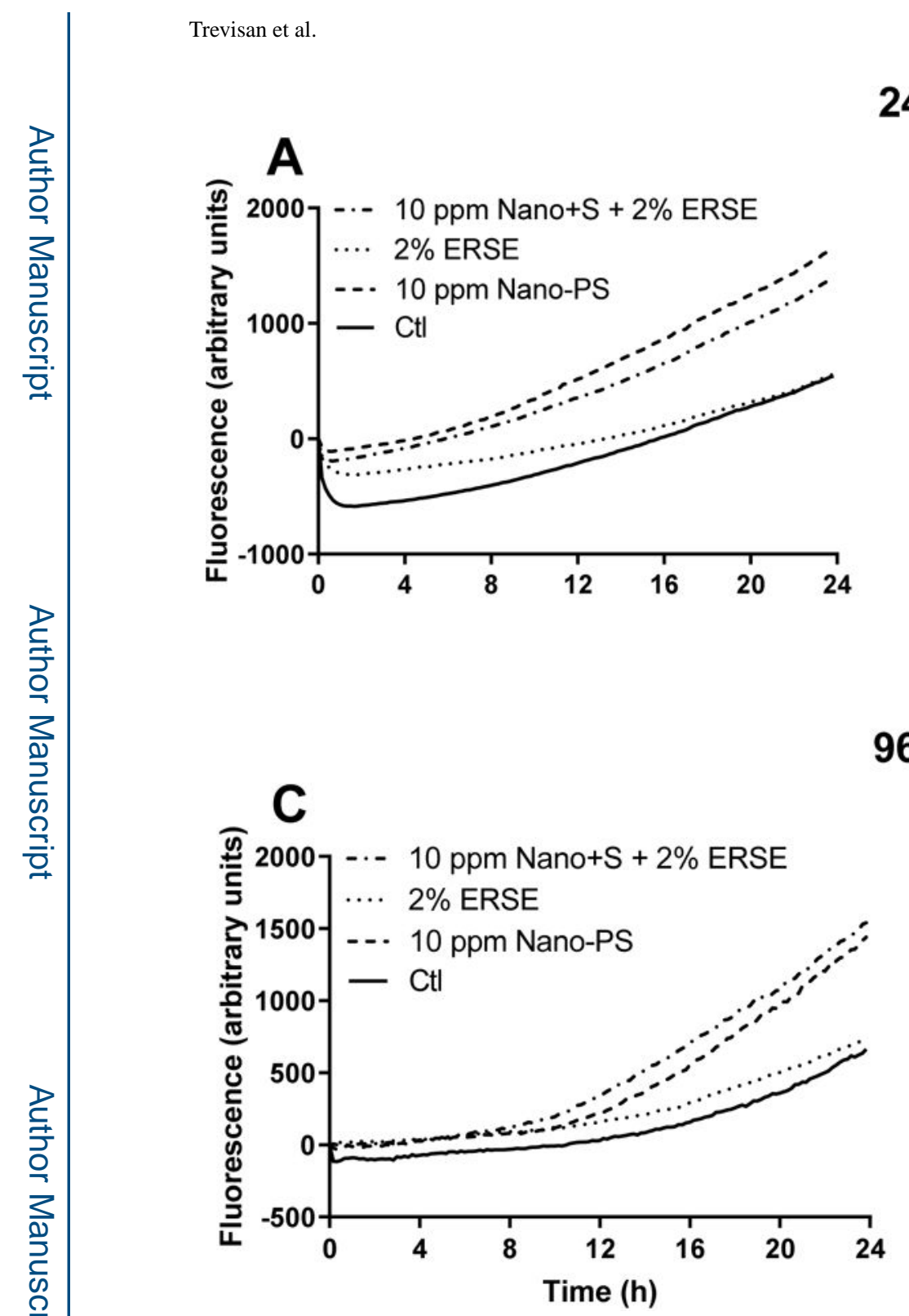

$24 \mathrm{hpf}$

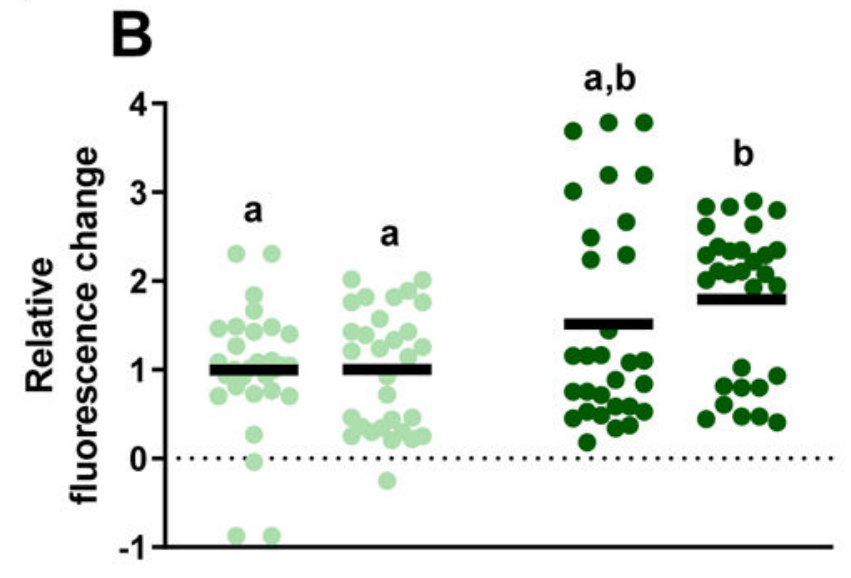

Figure 6:

NADH production in zebrafish embryos or larvae exposed to $44 \mathrm{~nm}$ nanopolystyrene particles (Nano-PS) in the presence of a real world environmental PAH mixture (Elizabeth River Sediment Extract - ERSE). Embryos were co-exposed at 6 hours post fertilization (hpf) to $10 \mathrm{ppm}$ Nano-PS and 2\% ERSE. NADH production was assessed in vivo over 24 hours, starting at 24 or $96 \mathrm{hpf}$ using the alamar blue assay. Changes in absolute fluorescence values are shown on the left ( $\mathrm{A}$ and $\mathrm{C}$, single representative experiment), and the relative fluorescence changes (fold change at the end of the assay, normalized by the control group) are shown on the right (B and D). Results are presented as scatter plot $(n=30)$ and mean (black bar). Data were analyzed by two-way ANOVA followed by Tukey's posthoc. Groups with different letters are significantly different from each other $(\mathrm{p}<0.05)$. 